\title{
SIDH: A Game-Based Architecture for a Training Simulator
}

\author{
P. Backlund, H. Engström, M. Gustavsson, M. Johannesson, M. Lebram, and E. Sjörs
}

In GaMe Lab Reasearch Group, School of Humanities and Informatics, University of Skövde, 54128 Skövde, Sweden

Correspondence should be addressed to P. Backlund, per.backlund@his.se

Received 24 June 2008; Accepted 19 December 2008

Recommended by Rafael Bidarra

Game-based simulators, sometimes referred to as "lightweight" simulators, have benefits such as flexible technology and economic feasibility. In this article, we extend the notion of a game-based simulator by introducing multiple screen view and physical interaction. These features are expected to enhance immersion and fidelity. By utilizing these concepts we have constructed a training simulator for breathing apparatus entry. Game hardware and software have been used to produce the application. More important, the application itself is deliberately designed to be a game. Indeed, one important design goal is to create an entertaining and motivating experience combined with learning goals in order to create a serious game. The system has been evaluated in cooperation with the Swedish Rescue Services Agency to see which architectural features contribute to perceived fidelity. The modes of visualization and interaction as well as level design contribute to the usefulness of the system.

Copyright () 2009 P. Backlund et al. This is an open access article distributed under the Creative Commons Attribution License, which permits unrestricted use, distribution, and reproduction in any medium, provided the original work is properly cited.

\section{Introduction}

The firefighter profession is stressful and dangerous, exposing its practitioners physically and psychologically highly demanding tasks in extreme environments [1]. One of the most hazardous tasks is to enter a building on fire and to search for victims. This activity is referred to as breathing apparatus entry (BAE) and requires a systematic and thorough scanning of the building where the sight may be extremely limited due to smoke. This in combination with the heat from fires forces the firefighters to hold a low position. When a victim is found, he/she must be dragged to a safe environment before the search can continue. The training for BAE is traditionally carried out in training areas with buildings of different types where victims are replaced with dummies. These methods have been shown to be effective but they are not optimal for all situations. First of all, they are relatively costly as they require trained instructors and access to a dedicated training area where each type of environment (e.g., hotel, ship, train, gas station, etc.) requires a separate physical model. If the same persons participate in repeated exercises they are likely to become familiar with these models. Simulator training solves the problem of students getting familiar with the physical models as new virtual models are fairly easy to create. Furthermore, as live training sessions are costly they need to be well prepared to create high value. Hence, virtual environments form an effective and efficient complement and preparation for real-world training [2] in order to increase its quality.

Simulators form a class of systems which emulate the operational environment, often by means of visual representations. Various game-based training systems are sometimes referred to as "lightweight" simulators [3]. Alexander et al. [3] identify a number of benefits with "lightweight" simulators and the use of video game engines, some of which are flexible technology, economy, and user control. The system presented in this paper extends the concept of gamebased training systems by adding multiple screen view and a novel interaction mode. These features add to immersion and increase the feeling of "being there". One issue in relation to all sorts of simulator training, and in particular concerning game-based simulators, is the transfer of skills and abilities to the real operational environment. Fidelity refers to the extent which the virtual system emulates the real world and is hence expected to be an important aspect of the system.

SIDH is a game-based firefighter training simulator developed in cooperation between the University of Skövde and the Swedish Rescue Services Agency (SRSA). An important property of SIDH, which differentiate it from traditional simulators, is that it is game-based. Game technology, hardware, as well as software have been used to produce the application. More important, the application itself is 
deliberately designed to be a game. Indeed, one important design goal is to create an entertaining and motivating experience combined with learning goals. In this way, learning becomes self-motivating and the system may be used for off-hour training. SIDH also utilizes multiple screen view to make the player surrounded by the virtual world and a novel sensor-based interaction mode to put a physical load on the player.

The aim of this paper is to report on the architecture of a game based training simulator for BAE and how its fidelity is perceived by the users. By this we extend the knowledge of how game technology can be utilized for serious games purposes as well as the practical usefulness of such a system.

\section{Background}

In this section, we give a brief account for our view on the concept of serious games and serious gaming, that is, the activity of using games for purposes other than entertainment. We also summarize previous work on firefighter simulations and give a brief background on Swedish firefighter training.

2.1. Serious Games. Today, the term serious games is becoming more and more popular (see, e.g., $[4,5]$ ). The term itself is established, but there is no current singleton definition of the concept. Zyda [6, page 26] defines a serious game as follows: "a mental contest, played with a computer in accordance with specific rules, that uses entertainment to further government or corporate training, education, health, public policy, and strategic communication objectives." Furthermore, Zyda [6] argues that serious games have more than just story, art, and software. It is the addition of pedagogy (activities that educate or instruct, thereby imparting knowledge or skill) that makes games serious. However, he also stresses that pedagogy must be subordinate to story and that the entertainment component comes first.

In our work, we define serious games as games that engage the user, and contribute to the achievement of a defined purpose other than pure entertainment (whether or not the user is consciously aware of it). A game's purpose may be formulated by the game's designer or by the user her/himself. The desired purpose, that is, a serious game, can be achieved through a spectrum ranging from the mere utilization of game technology for nonentertainment purposes to development of specifically designed games for some nonentertainment purpose or the use and/or adaptation of commercial games for nonentertainment purposes, which means that also a commercial off-the-shelf (COTS) game, used for nonentertainment purposes, may be considered a serious game. We also propose that any combination of the above would constitute a feasible way to achieve the desired effect.

Serious games can be applied to a broad spectrum of application areas, for example, defense, government, healthcare, marketing and communications, education, and corporate and industry [4]. A question of interest concerns the claimed positive effects of such games, or of applications from related and sometimes overlapping areas such as elearning, edutainment, game-based learning, and digital game-based learning. In addition to obvious advantages, like allowing learners to experience situations that are impossible in the real world for reasons of safety, cost, time, and so on $[7,8]$, serious games, it is argued, can have positive impacts on the players' development of certain skills. We also note that some of these positive effects of gaming are not necessarily associated with any specific training or information objectives. As discussed by Mitchell and SavillSmith [9], analytical and spatial skills, strategic skills and insight, learning and recollection capabilities, psychomotor skills, visual selective attention, and so on, may be enhanced by playing computer games. Other reports [10] have also pointed out the positive effects on motor and spatial skills. More specific positive impacts have been reported, for example, by Enochsson et al. [11], who found a positive correlation between experience in computer games and performance in endoscopic simulation by medical students. The better performance of gamers is attributed to their threedimensional perception experience from computer gaming. The positive effects of games may hence be further utilized if we can identify the correct content and accurately exploit the user's experience as a driving force for developing serious games. Swartout and van Lent [12] identify the areas of experience-based systems and experience-based education as potentially benefiting from such a game-based approach. The general idea is to influence users by exposing them to some type of experience. Even though gaming is not a replacement for simulation, it may well serve as a complement in some regards [13]. In this sense we find the intersection between gaming and simulation to be an interesting one.

2.2. Immersive Visualization. There are several ways of visualizing virtual worlds in order to offer a more immersive experience to the viewer than can be achieved using ordinary computer displays. The choice of visualization system depends on in what purpose it will be used and, what resources in terms of time, space, and finances are available.

The term head-mounted display (HMD) is used on a category of visualization systems which presents a virtual world directly in front of the viewer's eyes, via one or two small displays. Used with a head tracking system the viewer is able to look at different parts of the world by turning his/her head. There are many different types of HMDs in the market, for instance helmets, goggles, and lightweight glasses. There are also a number of features which may be supported by an HMD device, such as stereoscopic vision and semitransparent displays. A common property of HMDs is, however, relatively low resolution and a small field of view.

A CAVE is an, usually supercomputer-based, environment for visualization of, and interaction with virtual models and worlds. The first CAVE Automatic Virtual Environment (CAVE) was developed by Electronic Visualization Laboratory [14]. The purpose was to build a system for scientific visualization, with high demands on performance [15]. The original CAVE had three walls, consisting of screens for rear 
projection, and measures $2.5 \times 2.5 \times 2.5$ meters. On the walls and the floor, stereoscopic scenes are presented for a viewer whose head movements are tracked in order to correct the projection in real time. The viewer is also able to interact with the virtual world, for instance, by using a special glove. Several variations of CAVE have been built since 1991. One example is the six-sided VR-CUBE which was built at the Royal Institute of Technology in Stockholm in 1998 [16]. Here the viewer is completely surrounded by video and audio from the virtual world-in all directions.

2.3. Virtual Environments for Firefighter Training. The SRSA is the government authority responsible for the training of fire and rescue operatives. All municipal fire and rescue services staff are trained and certified by the SRSA. BAE is one of the tasks a firefighter has to perform. It is of crucial importance that the firefighter can remain orientated with very limited or no vision in a building. Traditionally the firefighter students practice search methods with and without smoke in different physical buildings.

As firefighters have to handle extreme tasks there is a continuous need to develop training programs to prepare for such tasks [17]. There exist a number of reports of the use of virtual environments for firefighter training in the literature.

Tate et al. [2] present a study where a virtual environment training system was used to prepare shipboard firefighters for a mission. The virtual environment allowed users, equipped with an HMD device, to navigate in a 3D model of the ex-USS Shadwell fire research and test ship. A group of 12 trained firefighters participated in a study where their performance in a firefighting training mission was evaluated. Half the group was offered traditional mission preparation and the other half prepared using the virtual environment. The result of the evaluation showed that the second group had a better performance than the group using traditional preparation.

In a different study, Julien and Shaw [18] present a virtual firefighter command training environment which allows its users to inspect a house on fire and to command virtual firefighters. The fire is extinguished by issuing the correct sequence of commands to the firefighters. Perdigau et al. [19] present an application for managing virtual reality scenarios and their main scenario is a firefighter training simulation used for crisis situations training and understanding.

There are also examples of commercial simulation software used for firefighter training. The tactical command trainer is a tool from VectorCommand Ltd. [20] which allows users to train emergency management.

2.4. Fidelity in Games and Visualization. Buy-in, that is, how a person recognizes that an experience is relevant for their training is related to the amount of fidelity and perceived realism. It is often thought that high fidelity and realism will increase the chances of buy-in. Alexander et al. [3] describe how realism can be perceived important even though it has no particular bearing on the task to be trained.

Fidelity and transfer are crucial factors in simulation training. The fidelity aspect implies that the necessary level of fidelity in a simulator must be compared with the training goals. A low level of fidelity can be sufficient when the simulation training is part of a larger context; whereas a high level of fidelity is needed when the training goals includes features that are too dangerous or to expensive to practice in real life [21]. Fidelity can be divided in to three categories: physical, functional, and psychological fidelity. Physical fidelity is defined as the degree to which the physical simulation looks, sounds, and feels like the operational environment in terms of visual displays, controls, and audio. Functional fidelity is defined as the degree to which the simulation acts like the operational equipment in reacting to the tasks executed by the trainee. Psychological fidelity is the degree to which the simulation replicates the psychological factors (i.e., stress, fear) experienced in the real-world environment, and engaging the trainee in the same manner as the actual equipment would in the real world [3].

A major issue concerning simulation training or gamebased training is about the absence of a surrounding context where the knowledge will be used. According to Alexander et al. [3], it is a fact that real environments create stress and excitement that cannot be recreated in a virtual environment. When it comes to learning new abilities it can some times be necessary to try to reach the same levels of stress and excitement in the simulator as in the "real" world. If we could reach this relationship it would be named as high psychological fidelity. Alexander et al. [3], also claim, in connection to this line of argument, that both negative stress and positive stress are shown to improve retain and transfer of knowledge from the virtual environment to the "real" world. In a study of game-based training and its effects on American soldiers made by Morris et al. [22], it emerged that soldiers who where exposed to stress during the military training performed better in a "real" situation, than the soldiers who where not exposed to stress. In fact the group that was exposed to stress got considerably higher points during the following practice. The higher points were due to the fact that they managed to administer time better and they also avoided enemy fire to a higher extent.

Concerning fidelity, there is a focus on what is needed in terms of complexity and graphic realism and how the users will be able to use the things they learn in the virtual reality situation in the "real" world. According to Linderoth [23], this means that fidelity and transfer covary. Within the fidelity element there is a relationship between functional, physical, and psychological fidelity. Functional and physical fidelity form the largest impact on the transfer element. Psychological fidelity is defined after how well the simulator can recreate the psychological factors, that is, stress and fear, which the user later will meet in the "real" world [3]. The psychological fidelity has a rather small amount of impact on the transfer element since it is a rather abstract element.

\section{SIDH: An Architecture for a Game-Based Simulator}

The main goal with SIDH was to develop a cave-based simulator game for training some aspects of BAE. The 


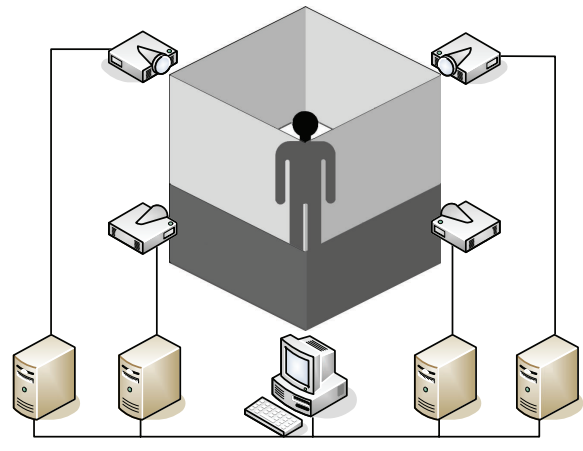

Figure 1: Schematic view of the cave.

project was carried out by expertise in game development and serious games, in close cooperation with expertise in firefighter training from SRSA. The game is not intended to replace conventional training, but rather to be used as a complement with focus on search strategies and ability to orient in unknown premises.

The game is to resemble real-world BAE in as many aspects as possible. This means that the player's task is to completely search different premises, with higher or lower complexity of the architecture, and rescue any persons inside. It should be possible to fill the premises with smoke, which, similar to real smoke, should be thinner close to the floor than close to the roof. The player should also be exposed to heat and physical and psychological stresses, which are characteristic parts of BAE. Furthermore, in respect of the educational purpose, it is crucial to record the player's actions during a game session for later analysis.

To reduce overhead time in the developing process, it was decided that the game was to be developed as a modification of a COTS game. In this way, the developers can focus on game content and specific features without first having to create an infrastructure from scratch. A feasibility study was committed in order to choose one of three common games-FarCry, Quake2, and Half-Life 2. All candidates were successfully modified to run in the cave environment. However, due to the realistic indoor environments and the ease of use of the level editor, Half-Life 2 was chosen as a platform for SIDH.

3.1. Cave Visualization. The cave is constructed from standard consumer electronics components. The purpose is to create a flexible environment for 360 degrees visualizations of, for example, games and films. The four walls are each $160 \mathrm{~cm}$ wide and $210 \mathrm{~cm}$ high. The upper $120 \mathrm{~cm}$ of the walls consists of screens for rear projection. Each screen is projected by a standard LCD projector, connected to a PC (Figure 1). These computers are interconnected in an Ethernet network together with another computer which acts as a server. All five computers are standard gaming PCs with regular graphics cards. The internal sound card of the server has been complemented with an external 5.1 sound card which delivers sound to four loudspeakers, one in each of the corners of the cave (Figure 1).
Half-Life 2 [24] is a multiplayer game which means that there is native network functionality for distributing information about the current state of the game objects. When playing a multiplayer game, one of the computers acts as a server responsible for controlling and synchronizing the progress of the game. When a server is running other computers in the network are able to connect and thereby become participating instances of the game running on the server.

When playing SIDH in the cave, the actual game, that is, the instance responsible for player input and game logic, is running on the server computer. The task of the fourclient-computers is to visualize the game running on the server, why the clients always have to be updated about the player's position and the state of the world. To achieve this, a native multiplayer feature called spectator mode is used. The original purpose of this feature is to make it possible for connected, but nonparticipating, clients to automatically follow and observe an active player. In that manner, the instances of the game running on the cave computers are observers, following the player on the server and hence showing the same view as the server without actually interacting with the game. The screens of the cave are however not to be clones of the server screen. Instead, they should present the world in four different directions, one direction for each screen, with the same origin as the server player. In the implementation, the game view is defined by a camera which acts as the eyes of the player (or the spectator). The position and orientation of this camera decides what is shown on the screen. By setting the orientation of a client's camera to a certain fixed direction, the client view will always show the world from that angle, regardless of the player orientation. Each view is thus rotated 90 degrees with respect to the adjacent screens. To produce the final 360 degree projection in the cave, the field-of-view (FOV) for the cameras was adjusted to 90 degrees. This is important since two adjacent views will overlap with a greater FOV value, and with a lesser value all parts of the world will not be visible at the same time.

3.2. Interaction Model. One of the goals with SIDH has been to make the player's interaction with the game as natural as possible. The player has to be able to move and look around in the virtual world, crouching as well as in a raised position. Moreover, it has to be possible to pick up and drop victims and control the game flow.

3.2.1. Navigation. One problem with navigating in a cave environment is the lack of obvious directions in terms of forward, backward, left, and right. Normally, when playing a first-person game, forward is defined by the center of the display, that is, the direction in which the player is currently looking. Pressing the forward key on the keyboard will always move the player in the desired direction since the world is rotating around the player and the keyboard. In the cave, however, it is not possible to use a single forward key since the directions are fixed to the real world. Hence, in order to decide what direction is forward for a cave player it is 

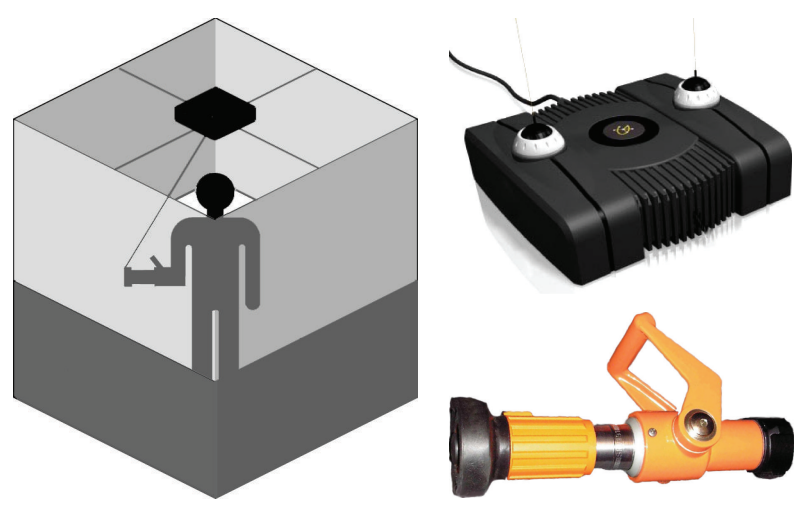

FIGURE 2: The string of the GameTrak (top right) is attached to the fogfighter nozzle (bottom right). N.B., only one of the GameTrak's [25] two controls is used.

necessary for the software to keep track of the player's current orientation. In SIDH, this problem is solved by using a GameTrak [25], which is an USB device working as a joystick with a string to pull as $Z$-axis. The GameTrak [25] is placed centered in the top of the cave, top down, with the string attached to the fogfighter nozzle (Figure 2). In this way, the nozzle's position in 3D space can be calculated. Since there may be some discrepancy between the direction expected by the player and the calculated direction (due to player deviation from the center of the cave), the calculated forward direction is visualized as a marker on the screens.

SIDH also calculates the nozzle's distance to the floor in order to decide whether the player is crouching (to increase the sight in smoke-filled areas) or not. Since a low position is depending on the player's height, the crouching threshold is dynamically adapted based on the highest nozzle position recorded during a mission. The information about the distance to the floor is also used to record so called markings. A marking is done by touching the floor with the nozzle, and is an action with different meanings in different phases of the game:

(i) to start the game in the beginning of a level,

(ii) to pick up found victims,

(iii) to drop rescued victims,

(iv) to confirm a completed search task.

3.2.2. Movement. For the player to be able to move inside the cave, a device named Stepometer has been developed. The idea with the Stepometer is that the player moves forward in the game by walking or running on the spot. The device consists of two accelerometer-based sensors which are attached to the player's feet, and a central unit processing the sensor signals and reporting steps to the server computer. The central unit is based on the Atmega8 microprocessor which, among other advantages, has native support for RS232 serial communication. The Stepometer is using this protocol over Bluetooth for wireless communication with the server computer.
A physical-based model is used to compute the speed, $v$, of the player. The updating of player speed is handled in the main loop of the game and the delay since last update is given by $d t$. For each update, the algorithm determines if the player has taken a step since last update, denoted with $s$. With $s=1$ the player has taken a step and with $s=0$ no step has been taken. The Stepometer has a sensor for each leg and the player has to alternate between left and right leg for the steps to be recorded. For each recorded step, the player speed is increased with an impulse, $I_{\text {step }}$, which is computed based on the players speed:

$$
I_{\text {step }}=I_{\max }-k_{1} * v \quad \text { where } k_{1} \text { is a constant. }
$$

This means that speed will increase quickly when the player starts to move, but as the speed increases each step will give a smaller and smaller contribution. The maximum speed of a player is hence given by $I_{\max } / k_{1}$.

The player speed is, in addition, decreased by a damping force, $I_{\text {damp }}$, proportional to the delay since the last step, denoted $t_{\text {prev }}$. The damping is given by

$$
I_{\mathrm{damp}}=t_{\mathrm{prev}}{ }^{2} * k_{2} * d t \quad \text { where } k_{2} \text { is a constant. }
$$

The quadratic nature of $I_{\text {damp }}$ implies that the damping is very low when steps are frequent but the damping grows quickly when the player stops moving. The speed is never allowed to become negative.

Combining the above, the speed is updated according to the following formula:

$$
v_{(t+d t)}=\max \left[0, s *\left(I_{\max }-k_{1} * v_{t}\right)+t_{\mathrm{prev}}^{2} * k_{2} * d t\right] .
$$

When the player is crouching the speed is divided by 2, which means that the step frequency has to be higher compared to when in a raised position, in order to keep up the speed. When carrying a victim, the speed is divided by 4 , why the step frequency has to be very high for the player to advance forward. This results in a significant increase of the physical strain, in analogy with carrying a victim in a real situation.

3.2.3. BAE Gear. Since the interaction is not depending on any fine motor activity, it is possible to let the SIDH player play the game using the same equipment as in BAE, that is, coat, trousers, gloves, boots, helmet, and air mask. Due to the look, feel, and smell of the equipment this may be used to increase the player's sense of realism. Since heat has a great influence on both physical and psychological stress during BAE, there was initially a discussion about placing infrared heaters on top of the cave. This would result in a realistic heat distribution which would force the player to crouch in order to decrease heat exposure. However, the computers and the projectors produced enough heat to make the room uncomfortably hot. This temperature and the fact that the equipment preserved the body heat (resulting from the player's physical effort) led to the decision that adding more heat would be a disadvantage concerning the entertaining aspects of the game. 
The air mask (Figure 3) is equipped with a radio receiver through which instructions from the virtual instructor is presented. To avoid interfering with the radio frequencies used by SRSA, the original radio system is replaced by two budget walkie-talkies. The other walkie-talkie works as a transmitter, which is connected to the internal sound card of the server. A slight modification of the software makes it possible to direct the voice of the instructor to this sound card, while all other sound effects are played back in the regular sound system.

3.3. Level Design. The levels for SIDH have been created in the Hammer world editor which is included in the Half Life 2 edition [24]. The Hammer world editor is a powerful tool. Apart from facilities for easy creation and texturing of elementary building blocks such as walls, floors, roofs, and ceilings the editor also gives access to a number of prefabricated models of humans, furniture, and other furnishing items. The prefabricated items have facilitated development work by reducing the number of specific models necessary to create for SIDH. Furthermore, the editor supports the manipulation of light, sound, smoke, and fire to alter environments.

The game flow is controlled by timers and triggers. Triggers are invisible objects which can be used to activate other objects or events such as, for example, a fire to flare or a new level to start. As an example, a trigger may be activated when the player approaches it. All levels in the SIDH game must have a specific setup of triggers and timers to handle the game flow. Important elements to game functionality are recycled in all levels, thus reducing the overhead when creating new tracks.

13 different levels (Table 1) have been developed in close cooperation with the SRSA. There is also a tutorial in which the player gets to learn the game and the interaction mode and two bonus levels. The game covers a variety of flats, warehouses, shops, and industry facilities to provide a relevant sample of environments for rescue personnel to train in. Each level provides new challenges with respect to size, complexity, and smoke. The difficulty is also increased by shorter time limits and the degree of harm made to the player by smoke and fire. Furthermore, the game provides challenges in terms of unexpected situations, such as victims hidden in closets and rooms difficult to locate. Other elements of stress are alarms, sirens, screaming victims, and explosions.

Each level starts with a female virtual instructor introducing the game task. The virtual instructor's voice is personified by an instructor from the SRSA, and the texture of the prefabricated models has been modified to resemble a real rescue personnel outfit. Each level ends in a debriefing room (Figure 4) where the player receives oral and visual feedback concerning his/her result.

A level is completed when all rooms and areas have been scanned and all victims rescued within time limit and with preserved player stamina. If the result is unsatisfying, the virtual instructor will inform the player of this and the player gets a new chance. The number of trials on each level is

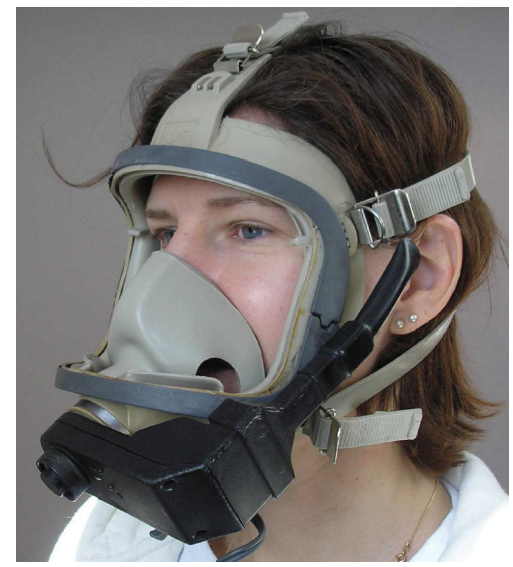

FIgURE 3: Air mask with radio unit.

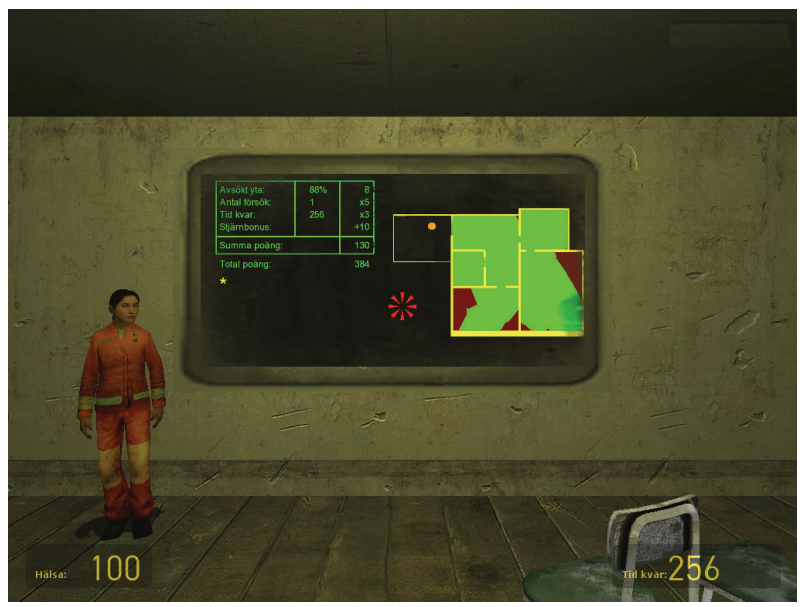

FIgure 4: The debriefing room. The board presents achieved score and an overview of the premises. The bright fields in the map represent areas visited by the player.

maximized to three in order to expose the player to a variety of environments.

When a level is successfully completed the player will get a score based on the proportion of the facilities that has been searched, time spent on the task, and whether it was the first, second, or third trial. If the area covered by the search exceeds $90 \%$, the player will be awarded a star bonus which will also generate a bonus score on the following level.

3.4. Source Modification. Besides implementing cave visualization and the interaction model, the source code had to be modified in order to implement required features and the specialized game flow. An example of such a modification is concerning the player's health level, which is constantly decreasing when acting in smoke-filled areas. When the player is in the room where the instructor is, that is, outside the hostile premises, the health level is recharged.

Furthermore, a supervising feature had to be implemented. SIDH is using the game engine's native functionality for recording metadata of a game to a file. This makes it 
TABLe 1: Game levels and their learning objectives.

\begin{tabular}{lll}
\hline Level & Description & Objective \\
\hline 0 & Tutorial & To handle the interaction model and game goals \\
1 & Two-room apartment & To get a first contact with the game \\
2 & Club building & To realize that time is limited \\
3 & Garage & To realize the advantage of crouching in smoke-filled areas \\
4 & Bonus level & To get a break \\
5 & One-room apartment & To look in closets \\
6 & Youth hostel & To handle large number of rooms \\
7 & Office space & To handle complex corridor architecture, closet \\
8 & Grocery store & To handle areas divided by shelves \\
9 & Three-room apartment & To handle heavy fire, closet, and limited time \\
10 & Large apartment & To handle extremely limited sight in complex architecture \\
11 & Basement storage area & To handle fenced areas filled with junk \\
12 & Four-room apartment & To handle doors at unexpected positions \\
13 & Hotel corridor & To handle numerous identical doors \\
14 & Butcher shop & To handle veiled areas \\
15 & Bonus level & To finish the game \\
\hline
\end{tabular}

possible to review the game exactly as it appeared on the screen when played. Hence, the feature works like screen capture except that the recording does not contain any image data, which requires a lot more storage space. The compact format allows SIDH to record every game without instantly filling the hard disk.

In addition to the native recording feature, a more specific logging functionality concerning the state and the activities of the player has been implemented. The player's position, orientation, and health level are logged ten times per second, and the markings are logged when committed. Together with information about the level geometry, this data is processed by an external tool which has been developed. This tool creates an orthographic image which shows not only what parts of the level the player has visited but also what parts actually have been seen. The algorithm for deciding the player's sight is based on information about the player's orientation, if there is smoke at the current position, the density of the smoke, and if the player is crouching or not. The image created is loaded into the game as a texture which is shown in the debrief room described above. The tool can also be used standalone to present an orthographic animation of the player's actions during the game.

It is worth mentioning that the modifications regarding interaction and cave visualization are implemented as additions to the original game features. Hence, it is possible to play SIDH on a single PC using standard input devices.

\section{Fidelity in SIDH}

This section reports on the results of an analysis of the perceived usefulness of the game as a training tool with respect to its fidelity. Data was collected from 32 students and one teacher during spring of 2008. The students participated in two questionnaires; the first one asked them about their expectations on simulator training and the second one captured their opinion after the simulator session. We observed training sessions for 11 students and carried out interviews with 10 students. Finally, the teacher in charge of the training sessions was interviewed. All data was compiled and we used a qualitative analysis approach to categorize it [26]. We focus on the aspect of fidelity even though complementary data was collected.

In the first questionnaire we asked a set of general questions concerning general data such as age and gender. We also asked the following questions (translated from Swedish). "How much do you play computer games on your spare time? How big is your experience from computer games? Do you think that it is possible to learn things from computer games? What is your prior knowledge of BAE entry? What is your prior knowledge of simulator training? In what ways do you think that simulator training can affect your performance in live exercises? In what other ways do you think that games and simulators can be used in your training?"

In the follow-up questionnaire: we asked the students questions about how their perception of the concept had changed. We also asked the following questions about their perception of the sessions and the usefulness of the simulator (translated form Swedish). "To what extent is fidelity important for the simulator to be useful? In which situations should simulator training be used? Would you consider using the simulator off-hours? What did you learn from your sessions? Did you learn anything which was useful during the live exercises? Do you think that the effect would be the same form playing in a single screen mode on a PC? What is the teacher's role during simulator training?"

The interviews were carried out following an interview guide which was adapted depending on the answers from the questionnaire. The questions concerned the participants' opinions on transfer (i.e., to what extent can knowledge from 
TABLE 2: Architectural features of SIDH.

\begin{tabular}{|c|c|}
\hline $\begin{array}{l}\text { Cave } \\
\text { visualization }\end{array}$ & $\begin{array}{l}\text { - Flexible system wrt screens and software } \\
\text { - Utilizing native functionality in software }\end{array}$ \\
\hline \multirow{5}{*}{$\begin{array}{l}\text { Interaction } \\
\text { model }\end{array}$} & - Fixed-based system \\
\hline & $\begin{array}{l}\text { - Track of current orientation and distance from } \\
\text { ceiling }\end{array}$ \\
\hline & - Stepometer with Bluetooth communication \\
\hline & - Physical-based model to compute speed \\
\hline & $\begin{array}{l}\text { - Original task equipment (including radio } \\
\text { communication) }\end{array}$ \\
\hline \multirow{3}{*}{ Level design } & - Utilizes native editor \\
\hline & - Timers and triggers to control game flow \\
\hline & - Levels with distinct learning objectives \\
\hline Source mod & $\begin{array}{l}\text { - Expanding native functionality to better suit the } \\
\text { training task }\end{array}$ \\
\hline
\end{tabular}

TABLE 3: Fidelity of SIDH features.

- Visualization and immersion by multiple screens

Physical - Fixed-based system navigation

fidelity - Physical strain

- Smoke behavior requiring low position

\begin{tabular}{ll}
\hline Psychological & - Stress \\
fidelity & - Time pressure \\
& - Resource management
\end{tabular}

Functional - Search strategies
fidelity

the simulator sessions be transferred to other situations?); fidelity (i.e., how did the participants perceive the simulator sessions with respect to the fidelity measures identified?), and motivation (i.e., in what ways can games and simulators affect motivation for training in a general sense?).

Fidelity is perceived to be a crucial part for the learning aspect and a high level of fidelity is necessary for the simulator to work in a proper way. We also claim that there is a connection between the construction of the simulator and what level of seriousness it will be perceived to have. One student argued that the higher the fidelity, the higher is the chance to learn something properly. Another student argues that a high level of fidelity supports the learning since it constitutes a chance to operate in a "close to real life" environment. In general, there was a positive perception of SIDH in terms of graphical appearance, sound, and user interaction. However, the teacher, being more conscious about the limitations of fidelity, was more inclined to see it as a complementary tool to live training. He highlights the fact that SIDH is useful to mediate search strategies for different types of environments.

The psychological strain was an important learning factor during the simulator training sessions. The students maintained that they learned how they, as individuals, react under stress and time pressure, and that the simulator sessions require concentration and presence of mind. Our findings also indicate that the simulator sessions require the person to be structured and calm during the minutes that they spend in the simulator. This is one important aspect of correctly carrying out a search strategy. Hence, our results indicate psychological fidelity in that the simulator recreates some of the stress and time pressure present in the original task. Game features, such as time pressure and pressure of saving lives, may hence be utilized to increase psychological fidelity.

One student suggested that simulator sessions should be used between the theoretical parts of the course and live training. He claimed that by doing this the student would be given a smoother transitional stage between the two parts. From a pedagogical perspective it makes sense that the students prepare for live training in order to increase its quality. This approach helps the students to get better results in live training since they got a chance to practice the procedure (i.e., technique for scanning a fire area) in virtual reality first. The majority of the students also claimed that the foremost thing they had learned in the simulator was a strategy for scanning a fire area.

The generation of smoke in SIDH has some resemblance to real smoke behavior [27]. The smoke will actually fill the room from the ceiling and down making it necessary to crouch in order to get a better view. Even though visually similar some of the participants commented negatively on the naturalness of the smoke claiming that sight was actually too good. One potentially negative aspect of this is that the students tend to trust visual stimuli at the expense of tactile stimuli, which is actually a poor strategy in many cases.

SIDH is a fix-based system with multiple screens which means that the virtual world is fixed and the player uses her/his sense of direction to navigate as opposed to a single-screen solution where the player sits still and the virtual world rotates. This feature, to some extent, adds to immersion. A majority of the observed participants felt a sense of misdirection and got lost.

The interaction mode of SIDH adds to the physical fidelity to some extent as game sessions become physically straining. The current version of SIDH does not utilize heat which would add to physical fidelity. However, as the participants wore full turn-out gear during the experiment some similarity to the real task was achieved. A majority of the participants experienced physical strain.

\section{Conclusion}

The main contribution from the presented work is a training simulator with good enough fidelity developed with relatively simple means. We have demonstrated the feasibility and usefulness of an architecture for a game-based immersive training simulator (Table 2). The novel interaction mode adds to immersion by introducing physical aspects into the game. We have also shown that inherent game functionality can be used and extended to create a variety of levels with distinct learning goals. Specific logging functionality concerning the state and the activities of the player has been implemented. This feature adds to the pedagogical usefulness of the system. 
SIDH is a firefighter training game, which, according to personnel and students of SRSA, is entertaining as well as effective regarding learning of the intended tasks [28].

One important aspect of the usefulness of a game-based simulator is the level of fidelity. It is important that the fidelity is in line with the intentions of how the simulator should be used. A reasonable level of fidelity is enough in situations where the (game-based) simulator constitutes a complement to other exercises. This is the situation with SIDH. Its main goal is to teach search strategies, that is, a demarcated part of the entire BAE task. Table 3 sums up how fidelity was perceived.

The focus on search strategies and the ability to orient in unknown premises were regarded as the most important gains from simulator training, due to the limited possibility of varying physical premises in the real world. Using the Hammer world editor to create premises for SIDH, the options are virtually unlimited.

\section{Acknowledgments}

The authors would like to thank the students and teachers at the Swedish Rescue Services Agency (SRSA) for their participation. Special thanks to Stefan Andreasson, Cecilia Hammar, and Marcus Toftedahl at the SRSA. This research was sponsored by the European Commission through the Interreg IIIC District project.

\section{References}

[1] J. Bos, E. Mol, B. Visser, and M. Frings-Dresen, "Risk of health complaints and disabilities among Dutch firefighters," International Archives of Occupational and Environmental Health, vol. 77, no. 6, pp. 373-382, 2004.

[2] D. L. Tate, L. Sibert, and T. King, "Using virtual environments to train firefighters," IEEE Computer Graphics and Applications, vol. 17, no. 6, pp. 23-29, 1997.

[3] A. L. Alexander, T. Brunyé, J. Sidman, and S. A. Weil, "From Gaming to Training: A Review of Studies on Fidelity, Immersion, Presence, and Buy-in and Their Effects on Transfer in PC-Based Simulations and Games," DARWARS, 2005, http://www.darwars.bbn.com/ downloads/DARWARS\%20Paper\%2012205.pdf.

[4] "Serious Games Initiative," June 2008, http://www .seriousgames.org.

[5] P. LoPiccolo, "Serious games," Computer Graphics World, vol. 27, no. 2, 2004.

[6] M. Zyda, "From visual simulation to virtual reality to games," Computer, vol. 38, no. 9, pp. 25-32, 2005.

[7] K. Corti, "Games-based learning; a serious business application," White paper, 2006, http://pixelearning.com.

[8] K. Squire and H. Jenkins, "Harnessing the power of games in education," Insight, vol. 3, no. 1, pp. 5-33, 2003.

[9] A. Mitchell and C. Savill-Smith, "The use of computer and video games for learning: a review of the literature," Tech. Rep., Learning and Skills Development Agency, Manchester, UK, 2003.

[10] A. Lager and S. Bremberg, "Hälsoeffekter av tv- och datorspelande. En systematisk genomgång av vetenskapliga studier," Tech. Rep., Swedish National Institute of Public Health, Östersund, Sweden, 2005.
[11] L. Enochsson, B. Isaksson, R. Tour, et al., "Visuospatial skills and computer game experience influence the performance of virtual endoscopy," Journal of Gastrointestinal Surgery, vol. 8, no. 7, pp. 874-880, 2004.

[12] W. Swartout and M. van Lent, "Making A game of system design," Communications of the ACM, vol. 46, no. 7, pp. 3239, 2003.

[13] B. Sawyer, "The serious games summit: emergent use of interactive games for solving problems is serious effort," Computers in Entertainment, vol. 2, no. 1, p. 5, 2004.

[14] Electronic Visualization Laboratory, 1991, http://www.evl .uic.edu/core.php? $\bmod =4 \&$ type $=1$ \&indi $=161$.

[15] C. Cruz-Neira, D. J. Sandin, and T. A. DeFanti, "Surroundscreen projection-based virtual reality: the design and implementation of the CAVE," in Proceedings of the 20th Annual Conference on Computer Graphics and Interactive Techniques (SIGGRAPH '93), pp. 135-142, Anaheim, Calif, USA, August 1993.

[16] Center for Parallel Computers, "The PDC Cube," 1998, http://www.pdc.kth.se/projects/vr-cube.

[17] R. Lasky, "Firefighter survival training: from reactive to proactive," Fire Engineering, vol. 157, no. 6, pp. 117-119, 2004.

[18] T. U. S. Julien and C. D. Shaw, "Firefighter command training virtual environment," in Proceedings of the Richard Tapia Celebration of Diversity in Computing Conference, pp. 30-33, Atlanta, Ga, USA, October 2003.

[19] E. Perdigau, P. Torguet, C. Sanza, and J.-P. Jessel, "A distributed virtual storytelling system for firefighters training," in Proceedings of the 2nd International Conference on Virtual Storytelling (ICVS '03), vol. 2897 of Lecture Notes in Computer Science, pp. 227-230, Toulouse, France, November 2003.

[20] Vector Command Ltd., February 2008, http://www. vectorcommand.com.

[21] E. Farmer, J. Rooij, J. Riemersma, P. Jorna, and J. Moraal, Handbook of Simulator-Based Training, Ashgate, Brookfield, Vt, USA, 1999.

[22] C. S. Morris, P. A. Hancock, and E. C. Shirkey, "Motivational effects of adding context relevant stress in PC-based game training," Military Psychology, vol. 16, no. 2, pp. 135-147, 2004.

[23] J. Linderoth, Datorspelandets mening-bortom idén om den interaktiva illusionen, Doctoral Dissertation, Göteborgs Universitet, Göteborg, Acta Universitatis Gothoburgensis, 2004.

[24] Valve Corporation, June 2008, http://orange.half-life2.com.

[25] In2Games Ltd., June 2008, http://www.in2games.uk.com.

[26] M. Q. Patton, Qualitative Research \& Evaluation Methods, Sage, Thousand Oaks, Calif, USA, 3rd edition, 2002.

[27] H. Gustavsson, H. Engström, and M. Gustavsson, "A multisampling approach for smoke behaviour in real-time graphics," in Proceedings of the Annual SIGRAD Conference, Skövde, Sweden, November 2006.

[28] P. Backlund, H. Engström, C. Hammar, M. Johannesson, and M. Lebram, "SIDH—a game based Firefighter training simulation," in Proceedings of the 11th International Conference on Information Visualisation (iV '07), pp. 899-907, Zurich, Switzerland, July 2007. 

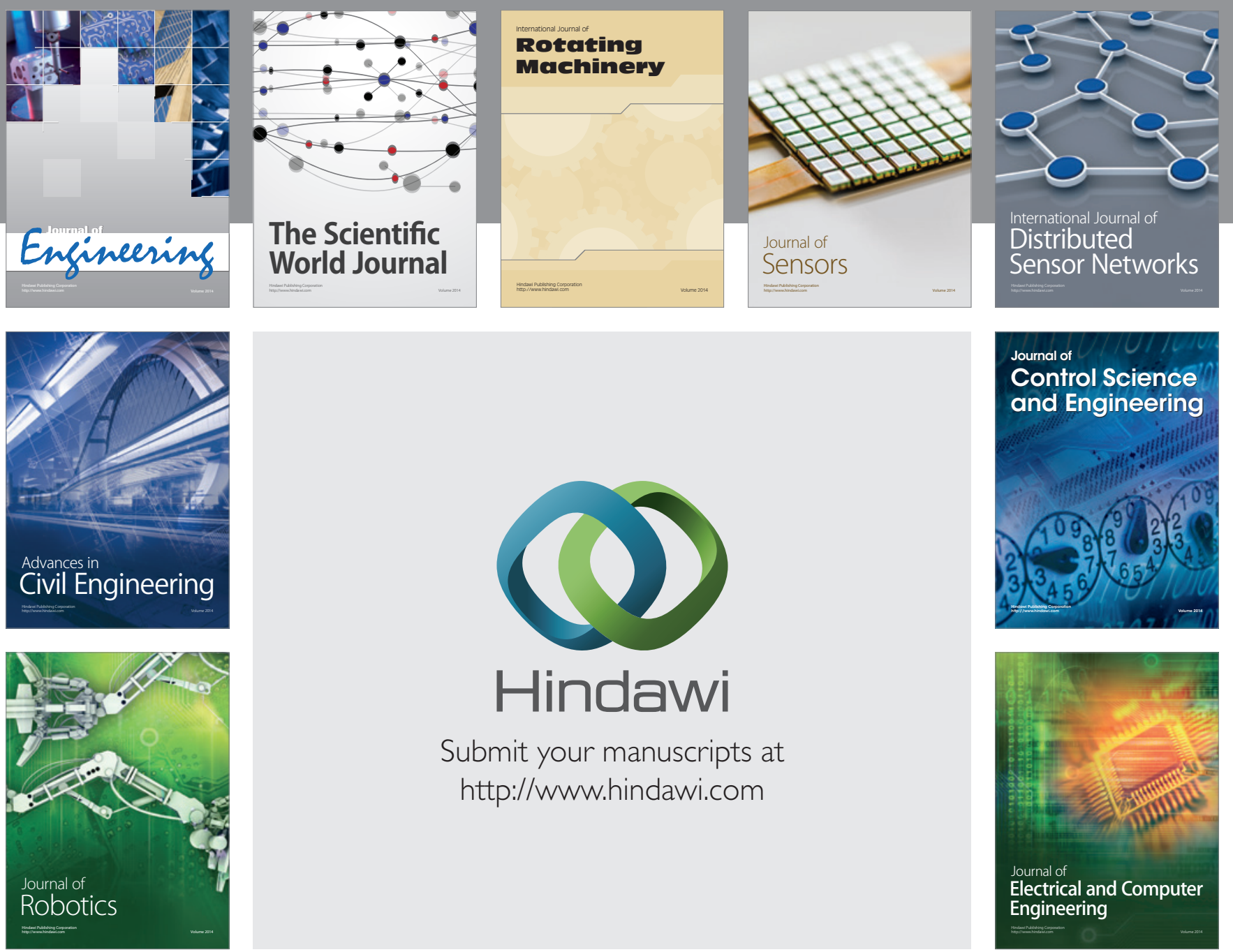

Submit your manuscripts at

http://www.hindawi.com
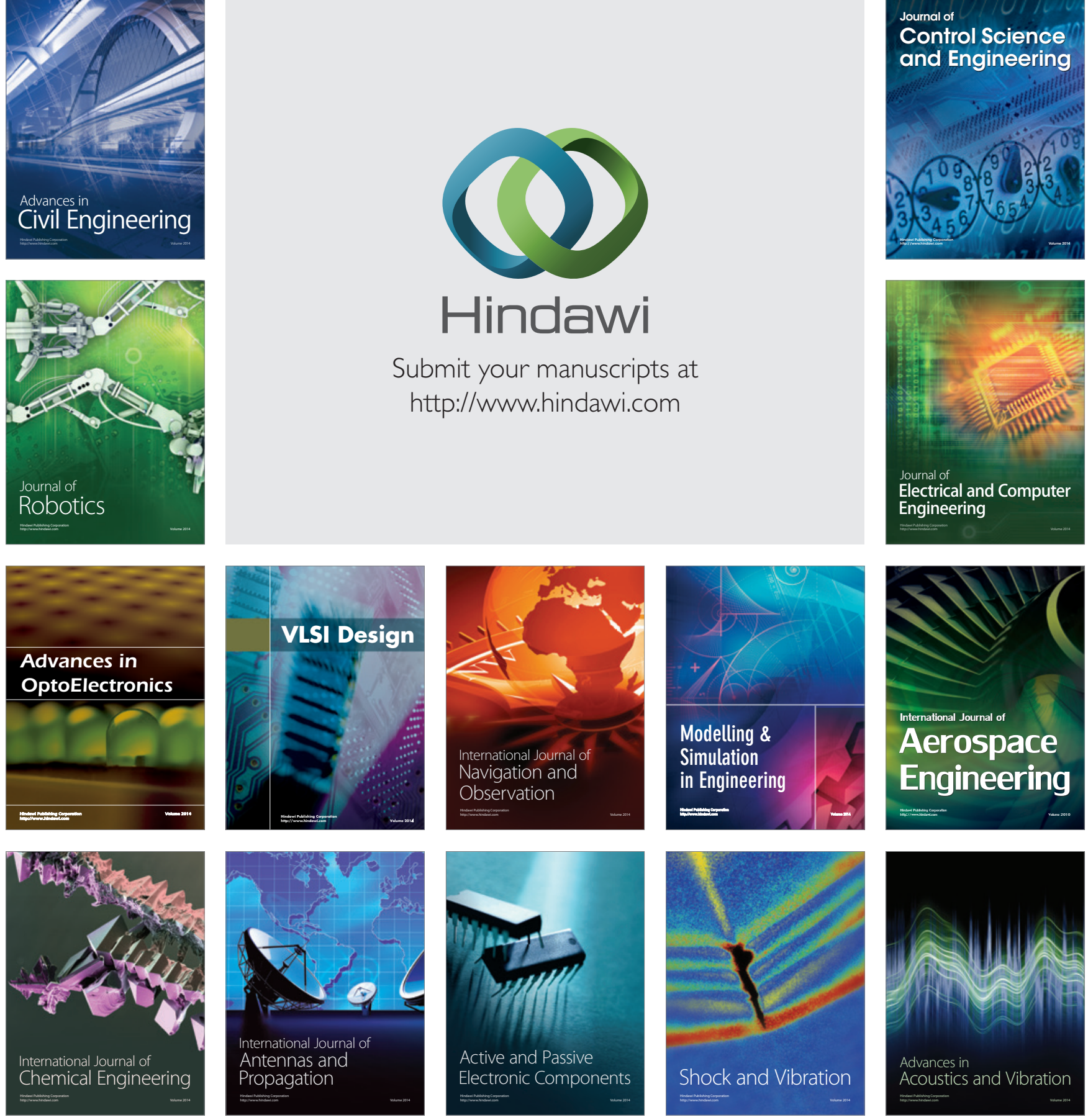